\title{
Hybrid Retrograde-Antegrade Recanalization of Acute Basilar Artery Occlusion
}

\author{
James D. Rossen ${ }^{a} \quad$ Edgar A. Samaniego ${ }^{b, c} \quad$ Mishelle Paullus ${ }^{c}$ \\ Santiago Ortega-Gutierrez ${ }^{b, c}$ \\ a Departments of Cardiovascular Medicine, Neurosurgery, and Neurology, ${ }^{b}$ Departments \\ of Neurology, Neurosurgery, and Radiology, and ' Division of Neurointerventional Surgery, \\ University of Iowa Carver College of Medicine, Iowa City, IA, USA
}

\section{Keywords}

Stroke $\cdot$ Basilar artery $\cdot$ Stent $\cdot$ Thrombectomy

\begin{abstract}
Acute basilar artery (BA) occlusion has a very poor prognosis. Recanalization can be challenged by bilateral vertebral artery (VA) occlusions, arterial dissection, or advanced atherosclerotic disease. We describe a case in whom the BA was accessed and recanalized through a retrograde-antegrade approach from the anterior circulation using a large posterior communicating artery (PCOM). Once the BA had been crossed retrogradely through the PCOM, another microcatheter was advanced antegradely through the VA into the BA and right posterior cerebral artery using the "buddy-wire" technique. In this way the BA was recanalized and reconstructed with stents. This technical note demonstrates a new approach to BA treatment when the antegrade access is hampered by advanced VA/BA disease or dissection.
\end{abstract}

(c) 2017 S. Karger AG, Basel

\section{Introduction}

Stroke due to basilar artery (BA) occlusion is a highly morbid event and outcomes may be improved by catheter revascularization. The standard antegrade transvertebral approach to BA occlusion recanalization may be hampered by vertebral artery (VA) obstruction, dissection, or tortuosity. An anterior-to-posterior approach to access the BA through the 

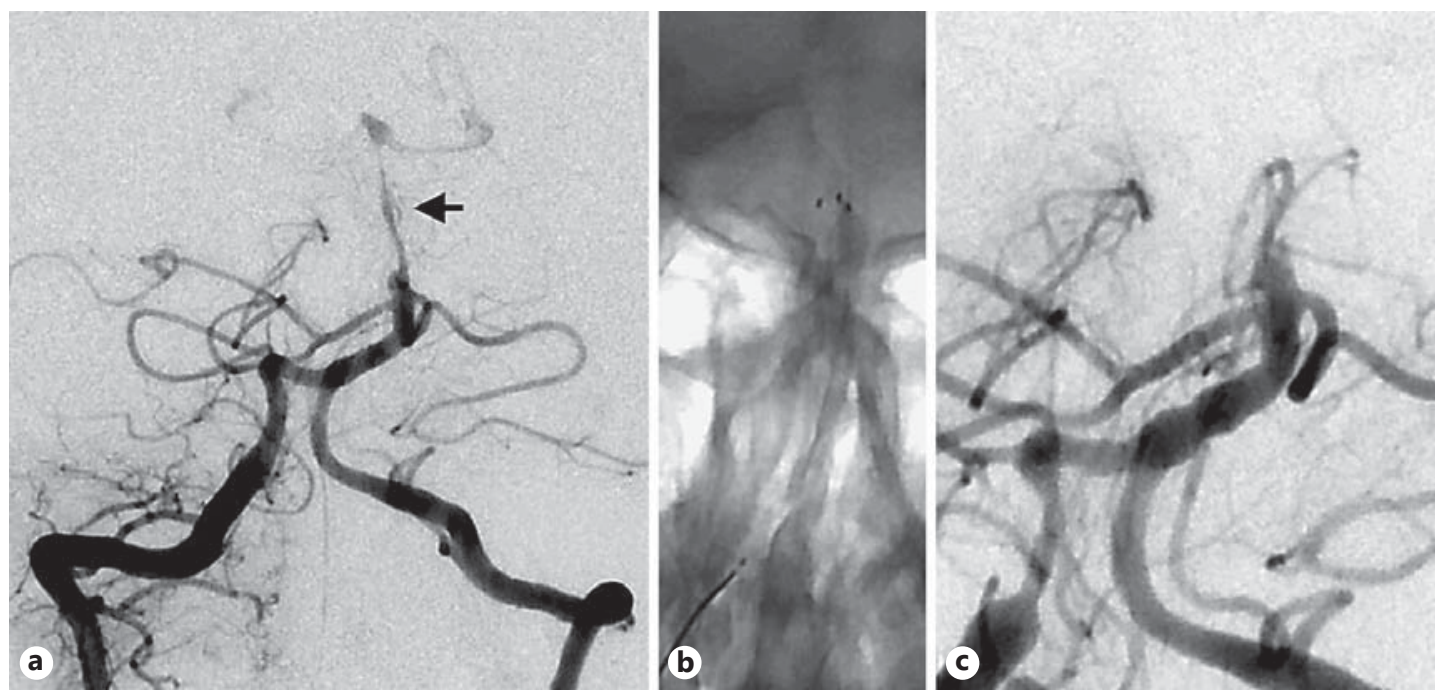

Fig. 1. a Right vertebral artery angiogram with posteroanterior cranial imaging demonstrating diffuse narrowing with subtotal occlusion of the basilar artery (arrow). b Deployment of the Solitaire $4 \times 20 \mathrm{~mm}$ stentriever. c Angiogram demonstrating proximal basilar artery occlusion after three thrombectomies.

posterior communicating artery (PCOM) has been described in treating aneurysms, thrombosis, and symptomatic stenosis [1,2]. We present the use of a combined anterior-to-posterior retrograde and antegrade approach to BA recanalization by stenting after failure of conventional antegrade technique.

\section{Case Report}

A 48-year-old right-handed man suddenly developed left-sided weakness and slurred speech while weight lifting. Local hospital telestroke assessment revealed a National Institutes of Health Stroke Scale score of 13 with right facial weakness, left arm and leg paresis, and severe dysarthria. A computed tomography scan demonstrated a hyperdense BA with no hemorrhage or brainstem effacement. Intravenous tissue plasminogen activator was started $1.5 \mathrm{~h}$ after the ictus and the patient was transferred for possible mechanical thrombectomy. Examination on arrival revealed minimal improvement in deficits with a National Institutes of Health Stroke Scale score of 9. A computed tomography angiogram confirmed an occlusion of the BA. The patient was referred for endovascular treatment, which was started $5 \mathrm{~h}$ after the ictus.

\section{Endovascular Procedure}

Under general anesthesia a 6-F MPD guide catheter was advanced into the V3 segment of the right VA. Angiography revealed severe diffuse narrowing of the BA with minimal filling of the posterior cerebral arteries (Fig. 1a). Antegrade recanalization of the BA was attempted using a Prowler Select Plus microcatheter (Medtronic, USA) and a Traxcess 14 microwire (Microvention, USA). However, neither the microcatheter nor the microwire would cross the distal BA into the posterior cerebral arteries due to the high atherosclerotic burden. Thrombectomy of the proximal BA was performed three times using a Solitaire $4 \times$ $20 \mathrm{~mm}$ (Medtronic) stentriever, but the BA remained occluded (Fig. 1b, c). We did not attempt to used another type of stentriever or a larger device since we thought that the main blockage was due to the presence of a plaque and/or arterial dissection.

A contralateral femoral artery percutaneous access was obtained and a 6-F MPD guide catheter was advanced into the right internal carotid artery. Angiography revealed a large right PCOM with brisk filling of the right posterior cerebral artery and terminal BA (Fig. 2a). An Echelon 14 microcatheter (Medtronic) and a Synchro 2 Soft microwire (Stryker, USA) were advanced from the right internal carotid artery to the basilar apex, traversing the PCOM (Fig. 2b). The microwire was advanced in a retrograde direction across the BA 

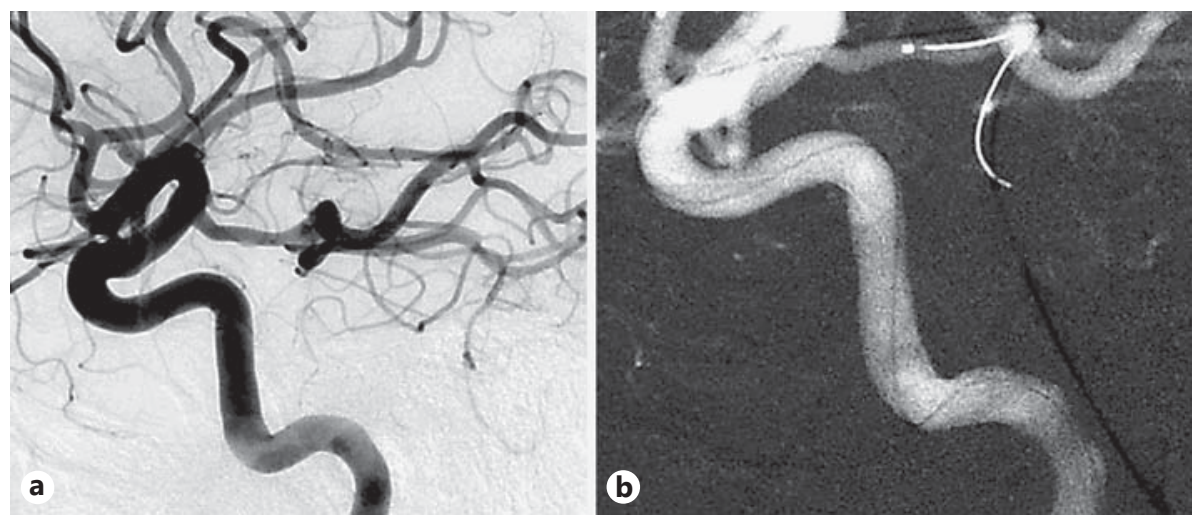

Fig. 2. a Sagittal right internal carotid angiogram demonstrating a large posterior communicating artery with filling of the right posterior cerebral artery and basilar apex. $\mathbf{b}$ Lateral roadmap demonstrating advancement of the microcatheter and the microwire from the right internal carotid artery to the posterior communicating artery and retrograde down the occluded basilar artery.
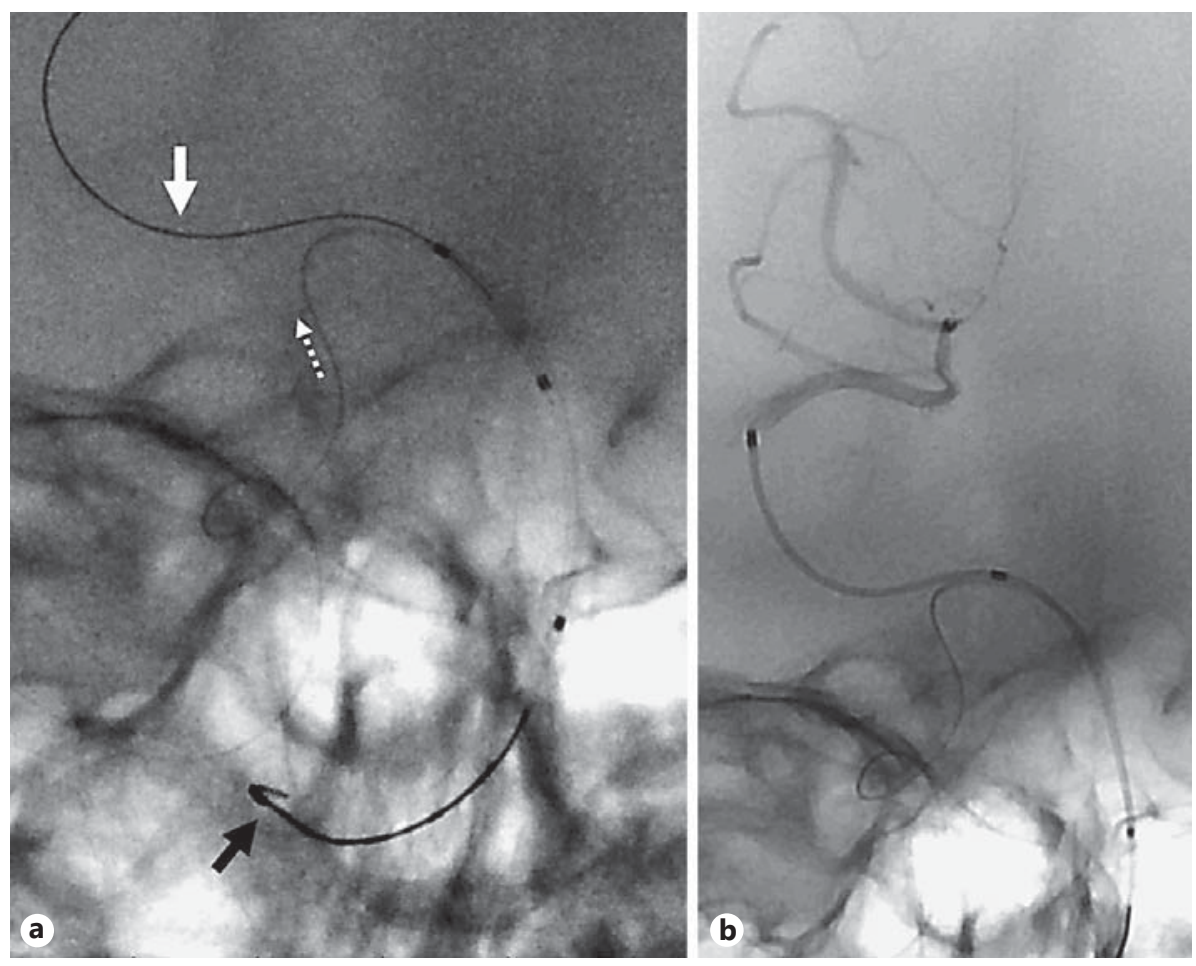

Fig. 3. a Cranial posteroanterior imaging demonstrating two microcatheters traversing the recanalized basilar artery. The retrograde microcatheter (black arrow) passes through the right internal carotid artery, the posterior communicating artery (dashed white arrow), as well as the basilar artery and terminates in the right vertebral artery. The antegrade microcatheter extends from the right vertebral artery to the right posterior cerebral artery (white arrow). b Right posterior cerebral artery antegrade microcatheter contrast injection confirming catheterization of the right posterior cerebral artery.

occlusion to the right VA and the microcatheter was advanced to the distal right VA. The antegrade microcatheter was advanced across the BA occlusion to the right posterior cerebral artery via the track created by the retrograde catheter using the "buddy-wire" technique and position was confirmed by microcatheter angiography (Fig. 3). VA injection revealed restoration of antegrade BA flow and evidence of extensive basilar 

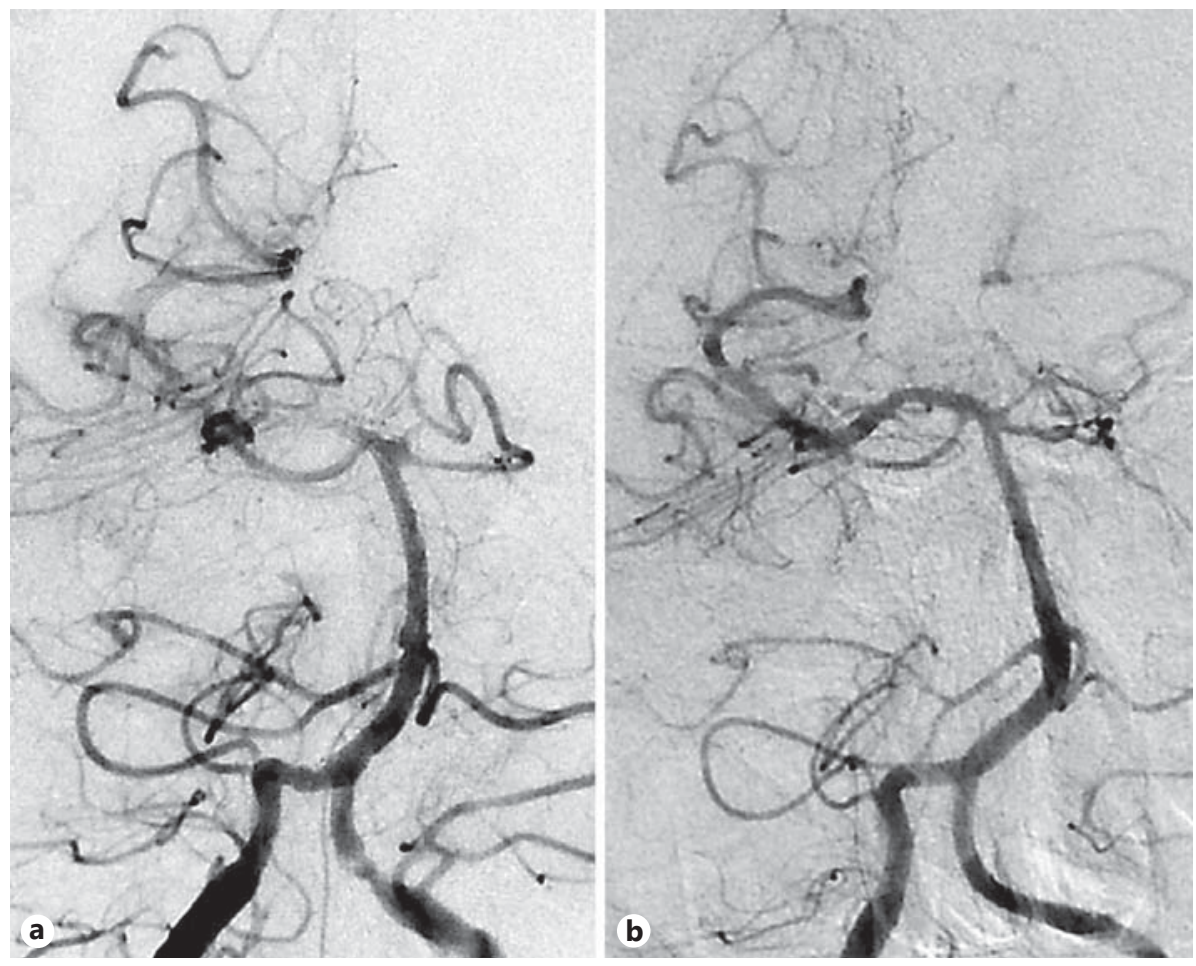

Fig. 4. Right vertebral artery injection with posteroanterior imaging immediately after basilar artery stenting (a) and at follow-up 6 months after treatment (b).

dissection. The antegrade microcatheter was removed using the long exchange wire technique and the BA was stented using Integrity $2.25 \times 8 \mathrm{~mm}$ and $2.25 \times 8 \mathrm{~mm}$ bare metal balloon expandable coronary stents. Despite the inherent risk of perforator occlusion after stenting with balloon-expandable stents, it was decided that the only way to maintain flow was reconstructing the BA. Final angiography revealed brisk antegrade filling of the BA and right posterior cerebral artery, with a fetal left posterior cerebral artery (Fig. 4a). Intravenous tirofiban was started before stenting and maintained for $6 \mathrm{~h}$. At the end of the procedure the patient was loaded with aspirin (325 mg) and clopidogrel (300 mg).

\section{Clinical Course}

Magnetic resonance imaging on hospital day 3 demonstrated infarction of the right hemipons and the left cerebellar hemisphere. Tracheostomy and percutaneous gastrostomy were performed on hospital day 7. The patient was discharged to a rehabilitation hospital on hospital day 21 with examination revealing hypophonia and left hemiplegia. Outpatient examination performed 17 weeks after the ictus revealed normal cognition, mildly dysarthric speech, no cranial nerve deficits, as well as left-sided mild proximal and severe distal weakness. He ambulated using a walker without assistance. Repeat cerebral catheter angiography at 6 months demonstrated wide patency of the BA and branches (Fig. 4b).

\section{Discussion}

Retrograde recanalization of coronary artery chronic total occlusions is an established technique [3]. It is useful because the distal plaque cap of a coronary occlusion may be more readily penetrated than the proximal cap. In this technique, both the occluded vessel and the vessel supplying collaterals to this vessel are cannulated with guide catheters. The distal segment of the occluded artery is accessed by traversing a collateral vessel with a guidewire and microcatheter. A channel is created from the distal to proximal segment through the true 
lumen or by intentional subintimal dissection and proximal segment reentry. Antegrade access across the new channel is established and the procedure is completed using balloon angioplasty and balloon expandable stenting.

This case report describes the use of a combined retrograde/antegrade technique to revascularize an intracranial artery total occlusion. Reported experience with a retrograde approach to cerebral artery obstruction is limited. Balloon angioplasty of a BA stenosis using an anterior-to-posterior approach via the PCOM in the setting of bilateral VA occlusion was reported by Chiam et al. [4]. Liu et al. [1] reported treatment of a BA occlusion with the Penumbra (Penumbra, USA) catheter aspiration system and intra-arterial tissue plasminogen activator thrombolysis via the PCOM. A combined retrograde/antegrade technique was used to revascularize a VA origin occlusion to allow antegrade aspiration thrombectomy of a BA occlusion [5]. A collateral originating from the ipsilateral thyrocervical trunk was traversed to enter the V3 segment and travel retrogradely to open the occluded VA origin. The newly opened VA was cannulated from the subclavian artery and thrombectomy was performed.

We believe our report to be the first to document use of a combined retrograde/antegrade approach for revascularization of an intracranial artery occlusion. The value of thrombectomy using conventional antegrade access in acute stroke is well established. An antegrade attempt was unsuccessful in our patient, perhaps because intimal dissection was the cause of the basilar occlusion, and we were not able to access the true arterial lumen. A large PCOM provided the path for retrograde recanalization of the true lumen which was accomplished easily. The BA obstruction was traversed in the antegrade direction with the retrograde system in place using a reverse "buddy-wire" technique. Basilar stenting was performed with balloon expandable stents because of extensive dissection with flow limitation.

We propose that a combined retrograde/antegrade approach to cerebral vessel recanalization be considered if antegrade technique is unsuccessful and persistent vessel occlusion is likely to cause a devastating stroke. This approach should be considered with prominent PCOMs that allow BA access. This report and previous literature support an aggressive approach in recanalizing the BA since complete occlusion is correlated with very poor outcome.

\section{Statement of Ethics}

The patient gave his informed consent for publication of his case.

\section{Disclosure Statement}

S. Ortega-Gutierrez is a consultant for Striker. E.A. Samaniego is a consultant for Microvention. J.D. Rossen and M. Paullus have no disclosures to make.

\section{References}

1 Liu W, Kung DK, Mahaney KB, Rossen JD, Jabbour PM, Hasan DM: Anterior-to-posterior circulation approach for mechanical thrombectomy of an acutely occluded basilar artery using the Penumbra aspiration system. World Neurosurg 2012;77:398.E17-E20.

2 Cross DT 3rd, Moran CJ, Derdeyn CP, Mazumdar A, Rivet D, Chicoine MM: Neuroform stent deployment for treatment of a basilar tip aneurysm via a posterior communicating artery route. AJNR Am J Neuroradiol 2005; 26:2578-2581.

3 Michael TT, Papayannis AC, Banerjee S, Brilakis ES: Subintimal dissection/reentry strategies in coronary chronic total occlusion interventions. Circ Cardiovasc Interv 2012;5:729-738.

4 Chiam PT, Mocco J, Samuelson RM, Siddiqui AH, Hopkins LN, Levy EI: Retrograde angioplasty for basilar artery stenosis: bypassing bilateral vertebral artery occlusions. J Neurosurg 2009;110:427-430.

5 Morales A, Parry PV, Jadhav A, Jovin T: A novel route of revascularization in basilar artery occlusion and review of the literature. BMJ Case Rep 2015;2015:bcr2015011723. 